\title{
Essential fatty acid metabolism in dogs and cats
}

\author{
J John E. Bauer, DVM, PhD, DACVN
}

College of Veterinary Medicine, Texas A\&M University, College Station, TX 77843-4474 USA

\section{Dietary Fats: Facilitative and Functional}

\section{Are there good fats and bad fats for dogs and cats?}

Although the concept of good fats and bad fats has been used in human nutrition, dogs and cats are not as susceptible to coronary artery diseases and can therefore consume greater amounts of saturated fats which are considered "bad" fats for humans. The reason for this is that dogs and cats have more good cholesterol (HDL) than bad cholesterol (LDL) no matter what types of fat they eat (1). Thus it is not advantageous to categorize different types of fats as either good or bad for these animals. Cats are likely similar to dogs in this regard although definitive data other than the fact that cats have high HDL cholesterol has not been obtained. In view of these metabolic differences, the author prefers to categorize the various types of dietary fats for dogs and cats as either 'functional' or 'facilitative'.

\section{Facilitative fats}

It is recognized that fat adds palatability and acceptable textures to food. Adding palatability demonstrates how fats can be facilitative because they help assure the intake of the necessary calories for well being. Beef tallow, primarily composed of saturated and monounsaturated fatty acids, is one of the most palatable fats for dogs, cats. Thus, tallow is not a bad fat for companion animals; instead it is facilitative. In dogs and cats, the amounts of tallow that may be fed without imposing a health risk would likely be detrimental to a human consuming a similar amount on a daily basis. Hence it is considered a 'bad' fat for humans; but merely facilitative for dogs and cats.
The saturated fat present in tallow is additionally facilitative for dogs and cats by contributing a high calorie fuel to the animal body providing energy to work, regulate body temperature, grow, reproduce, or simply survive. These fats are also facilitative because they can be stored in adipose tissues for future mobilization and used for energy when needed. Facilitative fats can be present in relatively large amounts in dog and cat diets without health risks except perhaps with respect to obesity where high fat diets, containing too many calories are overfed.

One additional way in which dietary fats are facilitative is because they assist the proper digestion and absorption of fat soluble vitamins from mixed micelles in the gastrointestinal tract. Because fats and fat soluble vitamins are insoluble in water, they must first be emulsified into smaller droplets by becoming dispersed with bile salts so that digestive lipases can break them into their digestible forms for absorption. Thus dietary fats facilitate the absorption of the fat soluble vitamins. In summary, a facilitative fat is one that has one or more of the following properties:

1.) adds palatability and acceptable texture to food

2.) is a dense source of dietary calories and energy

3.) promotes the absorption of fat soluble vitamins

4.) can be present in reasonably large amounts in dog and cat diets

Included in the facilitative fat category are dietary saturated fats such as palmitic and stearic, the monounsaturated fatty acid, oleic, and the trans fatty acids. These fats do not promote cholesterol elevations per se in dogs and cats as they do in humans. While 
they may be considered 'bad' for humans, they are simply facilitative in dogs and cats.

\section{Functional fats}

The first functional fat to be discovered was linoleic acid, an omega- 6 fatty acid. It was found to be an essential dietary component necessary for growth and prevention of skin lesions of dogs and other species. More recent studies have shown that both omega- 6 and omega- 3 fatty acids are essential. Both types can be converted to longer chain polyunsaturated fatty acids that have additional functions namely as precursors of the eicosanoids which are powerful physiological mediators of cell functions. These findings have added new complexities to the functional fatty acid category even though only modest amounts need to be included in the diet in order to meet tissue needs.

For the most part, the functional fatty acids that are also essential are all polyunsaturated by nature. Among this group are linoleic acid (LA), $\alpha$-linolenic acid (ALA), and under certain conditions docosahexaenoic acid (DHA) and arachidonic acid (AA). By contrast, conjugated linoleic acids may also fit into the functional category as new research is completed on their specific effects in companion animals. However, they do not have the requisite methylene interrupted sequence and thus are not essential. Also medium chain fatty acids are neither polyunsaturated nor essential but may be functional under some conditions. In summary, a functional fat is one that:

1. usually, but not always, an essential fatty acid or is derived from an essential fatty acid

2. participates in either an important structural and/or functional cellular process per se

3. or is converted to an important derivative that regulates cell function

\section{Definition of essential fatty acids}

The essential nature of a fatty acid is primarily due to an animal's inability to synthesize it in sufficient quantities to meet its metabolic needs. However, at the molecular level, important functional and structural characteristics both contribute to their essential nature. Functionally, the fatty acid must contribute in some significant way to health and well being. Structurally, it must contain at least two double bonds, the location of which must be in what is known as a methylene interrupted cis, cis-configuration. This precise molecular configuration enables the particular fatty acid to fold upon itself three dimensionally so that it can participate in cell membrane and physiologic events important for normal health. Once esterified into phospholipids these essential fatty acids significantly affect many membrane properties such as fluidity, compressibility, permeability, and fusion. Both omega-6 and omega-3 fatty acids meet these criteria.

Figure 1 shows a comparison of selected saturated, monounsaturated, and polyunsaturated fatty acids all of which have the requisite methylene interrupted sequence.

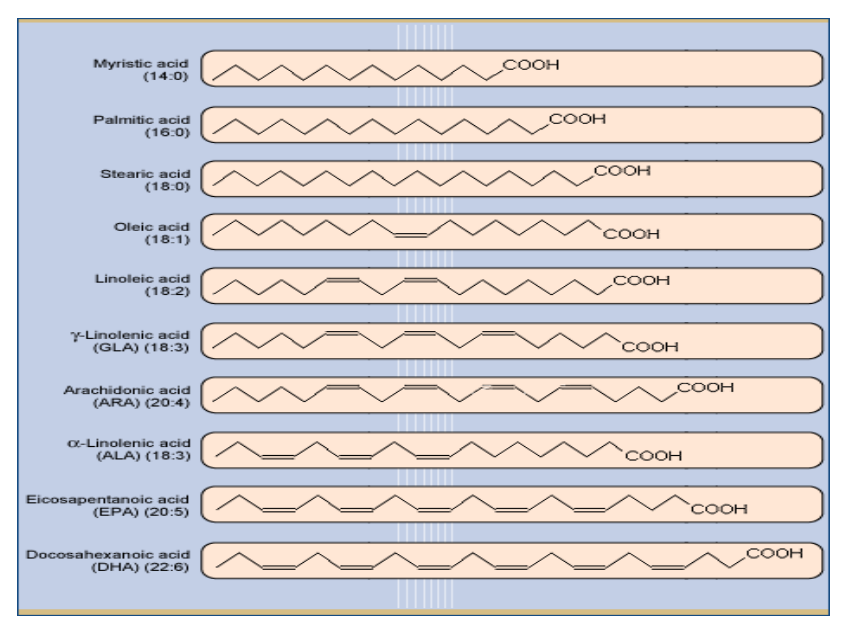

Figure 1 - Structural formulas of the saturated, monounsaturated, and polyunsaturated fatty acids also showing the methylene interrupted sequence of the polyunsaturated acids.

As a matter of contrast, conjugated linoleic acid, which contains two double bonds does not qualify as an essential fatty acid because its double bonds are "conjugated" instead of methylene interrupted. A comparison of the methylene interrupted sequence and the conjugated sequence of double bonds is shown in Figure 2. Both LA and ALA have methylene interrupted double bonds and are essential fatty acids. In addition, each serves as precursor of unique eicosanoids which are powerful physiological mediators of cell functions in numerous tissues. These derived long chain PUFA are also important because under 
some conditions or life stages, there may not be adequate conversion of the precursor 18 carbon acids making them conditionally essential.

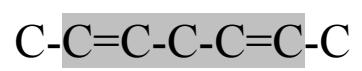

methylene-interrupted double bonds

$$
-\mathrm{C}-\mathrm{C}=\mathrm{C}-\mathrm{C}=\mathrm{C}-\mathrm{C}-
$$

\section{conjugated double bonds}

Figure 2 - Comparison of methylene interrupted fatty acid sequence (essential) and conjugated sequence (nonessential but potentially functional, e.g CLA, conjugated linoleic acid). Note the presence of the carbon atom between the double bonds in the methylene interrupted sequence.

\section{The Unique Fatty Acid Requirements of Cats}

\section{Omega-6 Fatty Acids and Adult Maintenance}

In the 1970s experiments on feline essential fatty acid (EFA) metabolism reported that domestic cats could not convert linoleic acid to arachidonate. This finding suggested that cats do not possess the necessary $\Delta^{6}$ desaturase to perform this conversion (2-4) (Figure 3). These observations were confirmed and extended by Sinclair et al (5) who concluded that while cats did not have significant $\Delta^{6}$ desaturase activity they did possess both $\Delta^{5}$ and a $\Delta^{8}$ desaturases. These and other authors $(6,7)$ thus proposed an alternative pathway to arachidonic acid synthesis in cats (Figure 3). Indeed subsequent studies found that when cats were fed diets rich in linoleic acid, both plasma and liver amounts of arachidonic acid were similar to when the diet also contained tuna oil (8). Thus cats appear to be able to synthesize arachidonic acid from linoleic acid at least to some extent and that an alternate pathway for its synthesis may exist. More recently, evidence of limited $\Delta^{6}$ desaturase activity in cat liver and brain has been confirmed by using sophisticated stable isotope techniques combined with gas chromatography and mass spectrometry (9) appearing when a diet completely devoid of arachidonate was fed. However, these data do not rule out the existence of the alternate pathway in cat tissues.

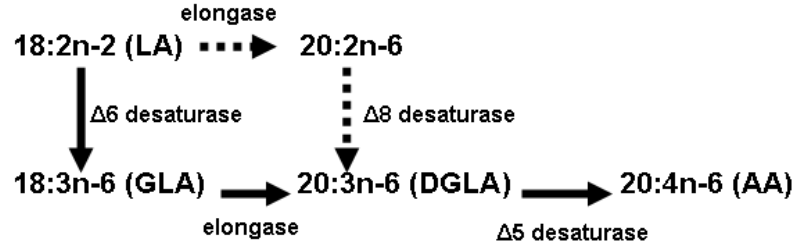

Figure 3: Pathways of synthesis of arachdonic acid from linoleic acid showing an alternate scheme involving $\Delta^{8}$ and $\Delta^{5}$-desaturation and by-passing the $\Delta^{6}$ desaturation step.

Omega-6 Fatty Acids, Growth, and Reproduction in cats

One study by Macdonald etal (10) found that male cats fed a linoleic acid deficient diet resulted in tubular degeneration of the testes. The fatty acid profile of testicular phospholipids had higher arachidonate concentrations when linoleic acid was present compared to the deficient group. By contrast, queens did not bear live kittens when fed the deficient diet. It was concluded that linoleic acid appears to meet the requirement for spermatogenesis but that arachidonate was necessary for reproduction in queens.

The need for dietary arachidonate for successful reproduction in queens was recently revisited by feeding one of three diets containing either $1 \%$ corn oil; $3 \%$ corn oil; or $1 \%$ corn oil plus $0.02 \%$ arachidonate before mating and throughout pregnancy (11). All animals became pregnant but a high incidence of congenital defects and low viability was found in the $1 \%$ corn oil group. By contrast, the diet containing 3\% corn oil without arachidonate supported reproduction. This study showed that queens are incapable of effective reproduction when maintained on a diet low in polyunsaturates including linoleic acid but that addition of small amounts of arachidonate restored this function. However, because the diet containing $3 \%$ corn oil without arachidonate also supported reproduction, it was concluded that other dietary factors may be involved. Of additional interest is that neonatal kittens from queens fed arachidonate in this study were found to also synthesize it from labeled linoleic acid precursor (12). 
Morris et al recently reported the effect of arachidonate depleted diets on both male and female feline reproduction (13). They confirmed their earlier finding that male cats fed diets containing linoleic acid, but not arachidonate, are fertile. In their study, 5 male cats fed hydrogenated vegetable oil containing diets devoid of arachidonate were mated with queens either individually or in small groups. The queens had been given commercial dry-type diets. Of the 13 queens mated, 12 conceived and with litter sizes ranging from 3 to 8 kittens. All kittens were observed to be clinically normal although of the 67 live kittens born 4 of them from 3 litters died after one day of age for unspecified reasons. Nonetheless the litter size exceeded the colony average of their laboratory using commercial diets. From this study, it was concluded that arachidonic acid is not an essential fatty acid for male cats for reproduction.

The reproductive outcome of 4 queens fed the hydrogenated vegetable oil diet all entered estrus, mated, and had subsequent body weight gains consistent with pregnancy (13). However, most of the kittens born live were eaten after birth with the proportion observed being much higher than the historical normal for this colony. Following this study, 2 of the queens were supplemented with $0.5 \mathrm{ml}$ of arachidonic acid and two were given $1.0 \mathrm{ml}$ once weekly for 10 weeks using a fungal derived oil containing $40.7 \%$ arachidonic acid and again bred. However, none of the queens conceived after this supplement. It was concluded that some other fatty acid(s) whether alone or in combination with aracidonate may be necessary for successful reproduction. Which fatty acid this may be is presently unknown.

\section{Omega-3 Fatty Acids in Cats}

The effects of vegetable-based $\alpha$-linolenic acid on reproduction of queens fed one of two levels of linseed oil (50 and $150 \mathrm{~g} / \mathrm{kg}$ diet) was compared to a safflower oil (50 g/kg diet)control diet (14) (3 queens per group). In the $50 \mathrm{~g} / \mathrm{kg}$ linseed group, the 3 queens gave birth to litters of 3-4 kittens. One queen had a second litter of 2 kittens both of which died. The other 2 queens did not have any further litters. In the $150 \mathrm{~g} / \mathrm{k}$ linseed oil group only one queen gave birth to one kitten which also died. Cats fed the linseed oil diets ultimately lost body weight. Their tissues were found to contain low concentrations of long chain omega- 6 acids and they developed signs of EFA deficiency. Because the limited feline $\Delta^{6}$ desaturase competes for both ALA and LA, high dietary ALA may preclude the conversion of linoleic acid to arachidonate. Hence excessive amounts of omega- 3 acids relative to omega- 6 fatty acids may be contraindicated in feline species.

Regarding the conversion of vegetable based omega-3 fatty acids to longer chain forms, adult cats were found to produce eicosapentaenoic acid (20:5n-3) and docosapentaenoic acid (22:5n-3) in liver and plasma and docosahexaenoic acid 22:6n-3 and 22:5n-6 in brain $(11,12)$. Of particular interest from these findings is that the final step of desaturation to form DHA appears to occur only in the nervous tissues, and not liver, of cats.

Independent of site of tissue synthesis, the important clinical question is whether the synthetic capacities of cats for the long chain omega-3 fatty acids are adequate under various life stages. Following their earlier study, Pawlosky et al. (12) fed diets with various amounts of corn oil and hydrogenated coconut oil prior to mating, during pregnancy, and subsequent lactation. Two reference diets also were evaluated containing AA and DHA. The corn oil diets were capable of maintaining AA concentrations in the developing retina and brain but only those diets containing DHA could support the high concentrations of DHA generally found in these tissues. Low concentrations of 22:5n- 6 were also found suggesting that kittens have a low capacity to produce this omega- 6 fatty acid as well as DHA. Differences in electroretinograms were observed in the LCPUFA deficient diet groups compared with control animals as an index of neural development.

The LCPUFA deficient diets of Pawlosky et al did not provide kittens with an adequate supply of $n-3$ fatty acids for proper accumulation of neural and retinal DHA during development and thus were inadequate for support of optimal visual function (12). Conversion of either the n-6 or n-3 18-carbon precursors may simply not occur to the extent needed in developing or immature cats. Finally, where practical diets are concerned, the presence of small amounts of dietary AA, EPA, and DHA (i.e., $0.14 \%, 0.02 \%$, and $0.03 \%$, as-is basis) in combination with high LA (i.e., $4.2 \%$ as is) resulted in insignificant conversion of ALA to 
LCPUFA when supplied as $0.88 \%$ (as is) in the diets of 19- to 20- month old cats (15).

\section{Summary and Conclusion}

Cats do not synthesize linoleic acid and require a dietary supply. Cats also have a limited capacity to synthesize arachidonic acid and an alternative pathway for it may exist. For maintenance needs, male, female, and neutered cats may be able to meet their requirements at very low levels of dietary inclusion of this fatty acid. A summary of the essential and conditionally essential fatty acids of cat is presented in Table 1. Specific recommended allowances for each life stage have been made as part of the 2006 NRC Nutrient Requirements for Dogs and Cats document from the National Research Council (16).

Table 1 - Summary of the Essential and Conditionally Essential Fatty Acids for Dog and Cat Life Stages.

\begin{tabular}{|c|ccc|}
\hline \multirow{2}{*}{ Nutrient } & \multicolumn{3}{|c|}{ Cats } \\
\cline { 2 - 4 } & Growth & Adult & Gest/Lact \\
\hline Omega-6 & $\mathrm{X}$ & $\mathrm{X}$ & $\mathrm{X}$ \\
LA & $\mathrm{C}$ & -- & $\mathrm{C}$ \\
AA & & -- & $\operatorname{Rec}^{\mathrm{b}}$ \\
Omega-3 & $\operatorname{Rec}^{\mathrm{b}}$ & -- & \\
ALA & -- & $\operatorname{Rec}^{\mathrm{b}}$ & $\mathrm{C}$ \\
EPA $^{\mathrm{c}}$ & $\mathrm{C}$ & & \\
DHA $^{\mathrm{c}}$ & & &
\end{tabular}

a) X, Essential fatty acid; C, Conditionally essential for the respective life stage.

b) Recommended but no requirement established

c) Many n-3 LCPUFA sources contain both EPA and DHA; EPA should not exceed $60 \%$ of EPA + DHA total.

Male cats may be able to synthesize enough arachidonate for reproduction. However, queens require an exogenous source of arachidonate for successful pregnancies and normal litters although for conception per se to occur arachidonate may not be needed.

For the omega- 3 fatty acids, high dietary amounts of $\alpha$-linolenic acid relative to linoleic acid may be contraindicated leading to EFA deficiency signs. As with the omega- 6 acids, adult cats can synthesize small amounts of long chain omega-3 acids from precursors. Nonetheless, in order to support the high retinal and nervous tissue concentrations of DHA needed for development, kittens may require this fatty acid as conversion of precursors may be insufficient to meet this need.

\section{Recent Studies on Omega-3 Fatty Acid Effects in Canine Species}

Several questions relating to omega-3 fatty acid metabolism have been studied in our laboratory. These include the following:

- Adults

- Can adult dogs convert $\alpha$-linolenic acid (ALA, 18:3n-3) to eicosapentaenoic acid (EPA, 20:5n-3) and docosahexanenoic acid (DHA, 22:6n-3)?

o Can ALA help improve skin and hair coat?

o Can adult dogs synthesize enough EPA to affect the inflammatory response?

- Gestation/Lactation

o Is ALA incorporated into canine milk?

o Is DHA present in canine milk when only ALA is fed?

- Puppy Growth

o Can puppies synthesize enough DHA to optimally support early neurological development?

o Is dietary DHA more efficient than ALA in this regard?

Skin and hair coat:

The first study we conducted compared supplementation of a complete and balanced commercial, dry-extruded type diet using whole ground sunflower seed (rich in omega-6 linoleic acid, LA) with whole ground flaxseed (rich in omega 3, ALA) (17). Two groups of dogs were fed a commerical diet supplemented with the respective ground oilseeds ( $3 \%$ 
by weight) for 84 days. Blood samples were obtained and plasma fatty acid profiles of phospholipid fractions were determined. The sunflower diet contained $9.3 \%$ of calories as LA and $0.4 \%$ calories as ALA while the flaxseed diet had $7.3 \%$ of calories as LA and $2.5 \%$ of calories as ALA. Results showed a rapid accumulation of EPA (at 4 days) reaching a steady state plasma concentration at 28 days. In addition, docosapentaenoic acid (22:5n-3, DPA) a DHA precursor was also found. However, no accumulation of DHA was seen. This study was the first to establish that EPA could be synthesized by dogs after ALA feeding although the amount of conversion appeared small. Additional questions remained, however, such as whether the derived EPA might help modify the inflammatory response or whether skin and hair coat benefits might exist .

Skin and hair coat condition scoring was conducted during the above study with improvements of skin and hair coat seen in both groups after 28 days.. However, differences due to diet were not seen and improvements were not sustained thereafter likely due to diet adaptation. Of particular interest, however, was that animals fed the flaxseed diet accumulated more LA than the sunflower group even though the sunflower diet contained more LA overall. We postulated that a sparing effect of ALA on LA with resultant skin and hair coat improvement may have occurred as well as the possbility of a total fat effect. Our later studies on skin and hair coat would observe a similar result.

A second skin and hair coat study compared 3 dry diets $(19,20)$. Diet A contained adequate amounts of EFAs ( $2.5 \%$ energy LA, $0.2 \%$ energy ALA) and dietary zinc $(120 \mathrm{mg} / \mathrm{kg})$. Diet B contained $8.8 \%$ energy LA and $0.2 \%$ energy ALA while Diet $C$ had $8.8 \%$ energy LA plus $1.6 \%$ energy ALA and increased zinc (both Diets B and C, $350 \mathrm{mg}$ zinc $/ \mathrm{kg}$ ). Total dietary fat of all diets was approximately 13\% (as-is); the diets were isocaloric at $3800 \mathrm{kcal} / \mathrm{kg}$ and formulated to be complete and balanced. Compared to a $9 \%$ total fat acclimation diet, all three diets improved skin and hair coat scores and the improvement was statistically significant after 7 weeks of feeding. The most dramatic differences seen were increased hair coat glossiness and softness. Fatty acid profiles of plasma phospholipids fractions again showed a sparing effect of ALA on LA.
The improvements observed appeared to be due, at least in part, to the higher total dietary fat concentrations of the diets compared to the acclimation diet rather than differences due to polyunsaturated fat types. Indeed, Diet A contained higher amounts of saturated fat compared to Diets B and C while dietary total fat was similar, yet hair coat scores were improved in all groups. Some additional benefits were seen with the polyunsaturated fat diets but the most dramatic skin and hair coat difference was due to total fat rather than fat type. It should be noted, however, that the appropriate balance of polyunsaturated fatty acids provides additional benefits beyond skin and hair coat. Thus these findings do not preclude their presence in modern commercial diets.

Of additional interest in this study were changes in the major lipid fractions from hair lipid extracts samples quantified after thin layer chromatography and densitometry with external standardization. Statistically significant increases in hair total cholesteryl ester (CE) concentrations were found in all three experimental diet groups at the end of the feeding period. It is unknown at this time whether this lipid alteration may correlate with skin and hair coat improvements. Nonetheless these preliminary data suggest a possible relationship when higher fat diets are fed. Should a positive correlation exist between these two parameters, hair lipid analysis may provide a useful, non-invasive technique to quantify dietary effects on skin and hair coat. Consistent with this possibility is that an earlier study also demonstrated increased sebum CE fraction in dogs fed diets containing increased total fat (21) Furthermore, our laboratory has previously shown that diets increased in total fat also elevate plasma $\mathrm{CE}$ in $\operatorname{dogs}(22)$.

\section{Inflammatory response:}

Long chain n-3 PUFA from fish oil or other marine sources seem to be especially capable of modifying inflammatory and immune responses. Diets containing only ALA as n-3 source or mixtures of ALA and fish oil may not perform as effectively in this regard when included on an equivalent weight basis (23-25) One reason for this is the inefficient rate of conversion of ALA to EPA (26). To confirm this possibility, a comparison of dietary ALA and EPA was conducted in our laboratory resulting in changes in 
neutrophil structure and function (232). In these studies, fish oil (i.e. containing EPA and DHA) outperformed linseed oil (i.e. containing ALA) at the same omega6/omega-3 ratio showing significantly different enrichment of EPA and DPA over AA, increased neutrophil membrane fluidity, decreased superoxide dismutase activities, and ex vivo phagocytosis. In addition, greater leukotriene $\mathrm{B}_{5}\left(\mathrm{LTB}_{5}\right)$ and lower leukotriene $\left(\mathrm{LTB}_{4}\right)$ production consistent with less inflammation were found. Another recent study described similar blunting of ex vivo neutrophil $\mathrm{LTB}_{4}$ production when dogs were fed a high n-3 PUFA diet using marine sources compared with corn oil $(\mathrm{mg} / \mathrm{kg}$ diet amounts of the n-3 PUFA not specified) (27). These findings are consistent with earlier reports that diets containing high marine source n-3 PUFA were particularly adept at modifying neutrophil and inflammatory skin responses of healthy dogs (24).

Taking this observation one step further, our work helped design dietary amounts of menhaden fish oil used by others in a study on its anti-inflammatory effects in dogs with osteoarthritis. Affected dogs were randomized to receive either a fish oil or control oil supplement. The amount of fish oil used was determined using predictive equations of fish oil enrichment as a function of dietary amounts developed in our laboratory (28). Dogs were fed their respective diets for 63 days and plasma and synovial fluid was collected and analyzed $(29,30)$. The fish oil diet resulted in significant increases of EPA and DHA in both plasma and synovial fluid with concomitant reduction of arachidonic acid. Also significant reductions in matrix metalloproteinase-2 and -9 (MMP-2 and MMP-9) activities which are known to contribute to cartilage destruction as well as a significant increase in a tissue inhibitor of the metalloproteinases (TIMP-2) were found. Furthermore synovial fluid bicyclo- $\mathrm{PGE}_{2}$ was significantly reduced with fish oil feeding. Of additional interest in this regard is the observation of Trumble et al in which synovial fluid $\mathrm{PGE}_{2}$ showed positive correlation with clinical variables of pain in dogs with osteoarthritis further (31) supporting the physiological significance of findings in these studies.

\section{Gestation, lactation and puppy neurological development:}

DHA appears to be necessary for neurological development (32). To test this idea, diets varying only in type/amount of PUFA were fed to female dogs as sole nutrition source beginning with estrus and throughout breeding, gestation, and lactation. Puppies were weaned to the same diets. Diets contained either low amounts of n-3 fatty acids, Lo n-3; moderate amounts of fish oil, Mod Fish; high amounts of fish oil, Hi Fish; or high amounts of vegetable n-3 fatty acids from flaxseed oil, Hi Flax. Mother's milk supplied exclusive nutrition to the puppies during suckling. Plasma phospholipid fatty acids during both gestation and lactation significantly reflected the diets fed and n-3 fatty acid dose responses were noted. However, dogs fed the Hi Flax diet showed no accumulation of DHA although EPA and DPA were increased as we have reported previously in non-parous adult dogs fed ALA (17).

Electroretinograms of puppies at 12 weeks of age revealed significantly improved visual performance in the Hi Fish group with superior rod response. Puppies in the Hi Flax group showed some improvement but not to the same extent as the Hi Fish group. In addition, this improvement occurred at a markedly high concentration of ALA compared to considerably lower amounts of fish oil (33). Thus preformed dietary n3LCPUFA vs ALA is a more effective means of enriching maternal plasma-DHA resulting in improved visual performance or puppies. Providing pre-formed long chain $n-3$ fatty acids in the diet as a means of enriching plasma appears to be conditionally essential especially for growth, development, and reproduction because slow and inefficient conversion of ALA to DHA may not be sufficient during these life stages.

Finally, it was of interest to observe that the fatty acid profiles of canine milk samples of dogs fed the $\mathrm{Hi}$ Flax diet showed enrichment only of ALA and not EPA nor DHA (34). Thus we were able to investigate whether puppies suckling this ALA-rich milk accumulated plasma DHA thereby suggesting its synthesis. The plasma phospholipid fraction of puppies during suckling showed not only the expected ALA and EPA enrichment but DHA was also increased compared to controls. After weaning, however, DHA content decreased while ALA and EPA remained elevated. This latter finding is similar to that seen in adult dogs (17). Thus, it appears neonatal canines may preferentially synthesize DHA from ALA at a time of life when demand for this fatty acid is especially high 
(i.e.during suckling) but only for a short time during this neonatal period. Again it is important to note that the milk from the Hi Flax diet was markedly enriched in ALA due to high diet amounts fed compared to the more modestly omega-3 enriched diets used in the fish oil groups. It is not known whether lower amounts of milk ALA would support similar DHA conversion. Hence the efficiency of including dietary DHA from fish oil for neonatal development is preferred to feeding ALA for both practical reasons as well as overall improvement of canine retinal development and response.

\section{References:}

Bauer, J.E. (2004) JAVMA 224:668-675

Rivers, J.P.W. et al, (1975) Nature 258:171-173.

Rivers, J.P.W., et al, (1976b) Proceedings of the Nutrition Society 35:69a.

Rivers, J.P.W. \& Frankel, T.L. (1980) In: Nutrition of the Dog and Cat, pp. 67-99 [R.S. Anderson, editor]. Oxford: Pergamon Press.

Sinclair, A.J. et al, (1979) Lipids 14:932-936.

Sinclair, A.J. et al (1981) British J Nutr 46:93-96.

McLean, J.G \& Monger, E.A. (1989) In: Nutrition of the Dog and Cat. Waltham Symposium Number 7. [Burger, I.H. \& Rivers, J.P.W. editors]. Cambridge, Cambridge University Press. pp. 349-342

Macdonald, M.L. et al, (1983) J Nutr 113:1422-1433.

Pawlosky, R. et al, (1994) J. Lipid Res 35:2032-2040.

MacDonald, M.L. et al, (1984) J Nutr 114:719-726.

Pawlosky, R.J. \& Salem, N., Jr. (1996) J Nutr. 126:1081S$1085 \mathrm{~S}$
Pawlosky, R.J. et al, (1997) Am J Clin Nutr 65:465-472.

Morris, J.G. (2004) J. Amim. Physiol. a. Anim.Nutr. 88:131137.

Monger, E.A. (1986) Polyunsaturated fatty acid metabolism in the cat. PhD Thesis, University of Melbourne, Australia.

Chew, B.P. et al, (2000) Role of Omega-3 fatty acids on immunity and inflammation in cats. In: Recent Advances in Canine and Feline Nutrition, Vol III. G.A. Reinhart, D.P. Carey, eds. Wilmington, Orange Frazer Press, pp 55-67.

National Research Council (NRC). 2006. Nutrient Requirements of Dogs and Cats, Washington, D.C., National Academy Press, pp 81-110.

Bauer J.E. et al, (1998) J Nutr 128:2641S-2644S

Rees, C.A. et al, (2001). Vet Derm 12:111-117

Kirby, N.A. et al, (2007) JAVMA 230:1641-1645

Kirby, N.A. et al, (2008) J An Phys An Nutr (in press)

Dunstan R.W. et al, In: Reinhart GA, Carey DP, eds. Recent Advances in Canine and Feline Nutrition, Vol III, Iams Nutrition Symposium, Wilmington, Orange Frazer Press. 2000;23-35.

McAlister K.G. et al, (1996) Vet Clin Nutr 1996;3,50-56.

Waldron, MK. (1999) Dietary fat effects on canine neutrophil membrane fatty acid composition and cell functions. Ph.D Dissertation, Texas A\&M University.

Vaughn, D.M. et al, (1994) Vet. Derm. 5:163-173.

Wander, R.C. et al,(1997) J. Nutr. 127:1198-1205.

Sinclair, A.J. (1975) Lipids 10:175-184.

Byrne, K.P. et al, (2000) Vet. Derm. 11:123-131.

Bauer, J.E. et al, (2002) J. Nutr. 132:1642S-1645S (2002).

Waldron, M. The role of fatty acids in the management of osteoarthritis. In: Clinical Edge. Advanstar Veterinary Health Communications. Lenexa, KS, October, 2004 pp 14-16.

Hansen R.A. et al, Proceedings, AOCS, Cincinnati, Ohio, 2004

Trumble, T.N. (2004) Am J Vet Res 65;1269-1275.

Waldron M.K. et al, (1998) JAVMA 213:619-622

Heinemann, K.M. et al (2005) J. Nutr 135:1960-1966.

Bauer, J.E. et al, (2004) J. Nutr. 134:2035S-2038S 